\title{
Nursing and medical students' perceptions of an interprofessional simulation-based education: a qualitative descriptive study
}

\author{
Woosuck Lee', Miran Kim², Yun Kang', Yu-Jin Lee', So Myeong Kim¹, Janghoon Lee², Soo-Jin Hyun', \\ Jihye $\mathrm{Yu}^{2}$ and Young-Suk Park \\ ${ }^{1}$ College of Nursing, Taegu Science University, Daegu and ${ }^{2}$ Ajou University School of Medicine, Suwon, Korea
}

Purpose: The purposes of this study were to describe the development and implementation of an interprofessional simulation-based education (IPSE) for undergraduate nursing and medical students, their perceptions of the impact of IPSE, and identify their changes in attitudes toward each other's health professions after participating in IPSE in South Korea.

Methods: This study used a qualitative descriptive design. A purposive sample of 43 third-year medical students and a convenient sample of 44 fourth-year nursing students participated in a 2-day IPSE program that consisted of ice-breaking and patient safety activities, and 4-hour three interprofessional team-based high-fidelity simulation education sessions. Data were collected through reflective journal after the IPSE program and keywords before and after the IPSE program, and were analyzed using the content analysis and word cloud analysis.

Results: Three themes emerged: "positive experience" with understanding roles and responsibilities and learning by doing in simulation environments being reported. In the second theme, "positive learning outcomes" participants reported enhancing collaboration and confidence in communication skills. The final theme "benefits to patients of interprofessional collaborative practice" included high quality of care and patient safety. Before the IPSE experience, most medical students perceived the nurse as nightingale and syringe, and nursing students perceived the doctor as order, expert, and knowledge. After their IPSE experience, both nursing and medical students viewed each other as colleagues.

Conclusion: These results suggest that the use of high-fidelity team-based simulation in IPE is effective in practicing and developing undergraduate nursing and medical students' interprofessional collaboration through hands-on experience.

Key Words: Nursing students, Medical students, Interprofessional education, Simulation-based education

\section{Introduction}

In today's constantly changing healthcare environment, patients have complex health-related needs and require collaborative health care to address these needs [1]. Interprofessional collaboration can improve care in patients with complex health-related needs, which positively affects their outcomes such as decreased length of hospital stay, increased satisfaction, and reduced healthcare costs [2-4]. Despite the benefits of interprofessional collaboration, healthcare professionals lack an understanding of their own and others' role and do not communicate effectively in providing health care $[2,5]$.
Received: August 8, 2020 • Revised: October 22, 2020 • Accepted: October 30, 2020 Corresponding Author: Yun Kang (https://orcid.org/0000-0001-9914-8969) College of Nursing, Taegu Science University, 47 Yeongsong-ro, Buk-gu, Daegu 41453, Korea Tel: +82.53.320.1752 Fax: +82.53.320.1761 email: ykang01157@tsu.ac.kr
Korean J Med Educ 2020 Dec; 32(4): 317-327.

https://doi.org/10.3946/kjme.2020.179

eISSN: 2005-7288

(C) The Korean Society of Medical Education. All rights reserved. This is an open-access article distributed under the terms of the Creative Commons Attribution Non-Commercial License (http:// creativecommons.org/licenses/by-nc/3.0/), which permits unrestricted non-commercial use, distribution, and reproduction in any medium, provided the original work is properly cited. 
Despite many factors contributing to interprofessional collaboration, the siloed approach in undergraduate health professions education and a lack of training in interprofessional collaboration are considered the foremost factor affecting interprofessional collaboration [6]. Thus, interprofessional education (IPE) among undergraduate health professions students is needed to practice and develop interprofessional competencies for future collaborative healthcare practice before graduation.

IPE in undergraduate health professions programs is the process where two or more professions students learn with, from and about each other to understand roles and collaboration, enhance communication, and improve patient outcomes $[2,7]$. In the United States, the Interprofessional Education Collaborative (IPEC) Expert Panel has developed the four core competencies necessary for effective interprofessional collaboration. These include value/ethics for interprofessional practice, roles and responsibilities, interprofessional communication, and teams and teamwork, which needs to be strengthened all together rather than as one of the core competencies of IPE [3,7].

Simulation education has recently become a favorable type of IPE in undergraduate health professions programs [8,9] and helps develop interprofessional teamwork and communication [2,10]. Interprofessional team-based simulation education can provide a realistic educational approach and standardized experience using simulation modalities and clinical scenarios designed to increase interprofessional collaborative practice $[11,12]$. It allows students from two or more health professions to practice together and then reflect on their collaborative practice such as teamwork and communication skills by creating clinical situations in a simulated environment [12]. The integrative review of IPSE studies suggests that interprofessional simulation-based edu- cation (IPSE) is well received by undergraduate health professions students and shows positive outcomes on both their interprofessional competencies such as interprofessional communication and teamwork and their affective behaviors such as increased self-efficacy and positive attitudes toward interprofessional learning [9]. Despite these positive findings, IPSE is still relatively new in Asian countries such as South Korea. The majorities of studies were conducted in Western countries such as the United States and Canada [9]. Thus, it is unclear whether the effects of the IPSE in Western countries are transferable to Asian countries due to different cultures. To our knowledge, this is the first study to develop and implement the IPSE for undergraduate nursing and medical students in South Korea. This study aimed to describe the development and implementation of IPSE for undergraduate nursing and medical students and explore their perceptions of the impact of the IPSE and their changes in attitudes toward each other's health professions after participating in the IPSE.

\section{Methods}

\section{Design}

This study used a qualitative descriptive design with reflective journal and keywords writings. A purposive sample of 43 (28 male students [77.8\%] and 15 female students [29.4\%]) of the 43 third-year medical students who were enrolled in second semester of third-year courses at Ajou University School of Medicine voluntarily participated in the IPSE. Considering the number of medical students, a convenient sample of 44 (eight male students [22.2\%] and 36 female students [70.6\%]) of the 295 fourth-year nursing students at 
Taegu Science University College of Nursing voluntarily participated in the IPSE.

\section{Interprofessional simulation-based edu- cation program}

The IPSE program proceeded through five phases: (1) agreement between Taegu Science University College of Nursing and Ajou University School of Medicine; (2) analysis on education condition; (3) IPSE program design and development; (4) IPSE program implementation; and (5) IPSE program evaluation (Fig. 1).

1) Educational exchange agreement between Taegu

Science University College of Nursing and Ajou

University School of Medicine

Leadership at both universities perceived the importance of the interprofessional collaboration and their students' IPSE experience in undergraduate health education programs, so that they can perform interprofessional collaboration when they enter the workplace. Thus, we decided to provide IPSE opportunities for our nursing and medical students.

Six months prior to IPSE program implementation, we signed an educational exchange agreement to prepare for IPSE program, considering the academic schedule of both groups of students. We identified and adopted the framework of the IPEC core competencies to develop IPSE scenario template $[3,7]$. We met to discuss how to implement the interprofessional team-based highfidelity simulation (HFS)-based education and then formed three teams (i.e., two nursing and two medicine faculty members per team) to develop three modules.

\section{2) Analysis on education condition}

We examined nursing and medical participants' simulation learning experience to develop IPSE program. Nursing student participants experienced three HFSbased courses and completed a 20-week clinical practicum for adult, child, women's health, mental health, and community health nursing. Medical student participants did not experience HFS-based courses and completed a 36-week clerkship for internal medicine,

Fig. 1. Development and Implementation Process of Interprofessional Simulation-Based Education Program

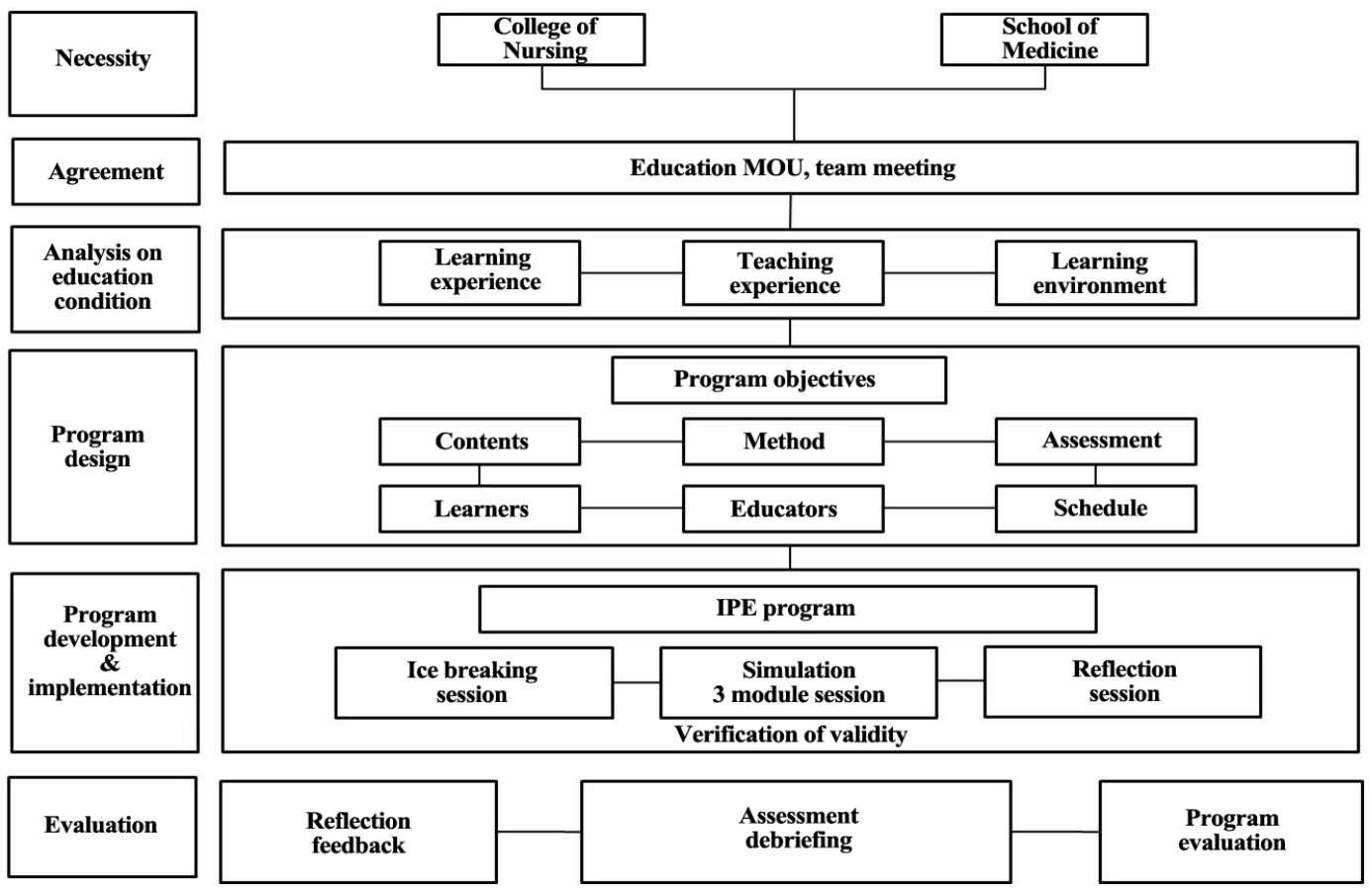

MOU: Memorandum of understanding, IPE: Interprofessional education. 
surgery, pediatric, obstetrics and gynecology, and psychiatry before this program. Both groups of students have not received formalized IPSE program.

Regarding nursing and medicine faculty facilitators' simulation experiences, all nursing faculty members have more than one semester of simulation experience. One medicine faculty member has simulation experience and the other five members had received formal training in simulation. Before implementing the IPSE program, a 1-hour facilitator training for the IPSE program was conducted. A guide for facilitators in each IPSE module included the learning objectives, plan, descriptions of scenarios, and debriefing methods, and was provided.

Considering adequate rooms and facilities to $\mathrm{ac}^{-}$ commodate a large number of nursing and medical students, faculty and staff, it was decided to implement the IPSE program at the nursing simulation center, Taegu Science University College of Nursing.

\section{3) IPSE program design}

The IPSE program specifically designed for undergraduate nursing and medical students consisted of introduction, ice-breaking and patient safety activities, three IPSE modules, and reflection sessions.

Each team discussed the learning objectives for their IPSE module that focused on interprofessional collaborative practice in clinical scenarios regarding deteriorating patients with acute myocardial infarction, and febrile seizure and postpartum bleeding after vaginal delivery. We made module learning objectives based on three domains of learning: knowledge, skills, and attitudes, considering the levels of fourth-year nursing students and third-year medical students. We developed the scenarios consisted of chest pain management in adult men with acute myocardial infarction, febrile seizure management in child, and postpartum bleeding management after vaginal delivery, using the IPSE scenario template. We then reviewed, discussed, and refined these scenarios within each module team until agreement was reached (Table 1). One student group consisted of two nursing students and two medical students, which resulted in 21 groups.

\section{4) IPSE program development and implementation}

The 2-day IPSE program was conducted from November 26 to November 27, 2019. One week prior to the IPSE program implementation, pre-study resources on three IPSE modules were given to the participants. The IPSE program began with the facilitators using introduction and ice-breaking activities to build a sense of community and shared purpose. Ice-breaking activities included puzzle games in which the student group had to find the missing puzzle piece in order to win the game. Patient safety activities were then provided to the student groups who had to identify risk factors in surgical or medical patients in simulated environments.

During the IPSE session, each student group was led by one nursing facilitator and one medicine facilitator. All participants in the groups took role-players and observers in turns. While one student group of four participants took the role play at one time, the next student group observed the scene in person. The IPSE session used a five-step model: pre-briefing, prescenario activities, task training, simulation running, and debriefing. Each IPSE module session took 4 hours and all participants in the groups participated in two IPSE module sessions.

\section{5) IPSE program evaluation}

At the end of the IPSE program, all participants gathered together in one place and had the time for a more thorough debriefing and reflection about their IPSE experience.

\section{Data collection procedure}

At the end of the IPSE program, all participants were 


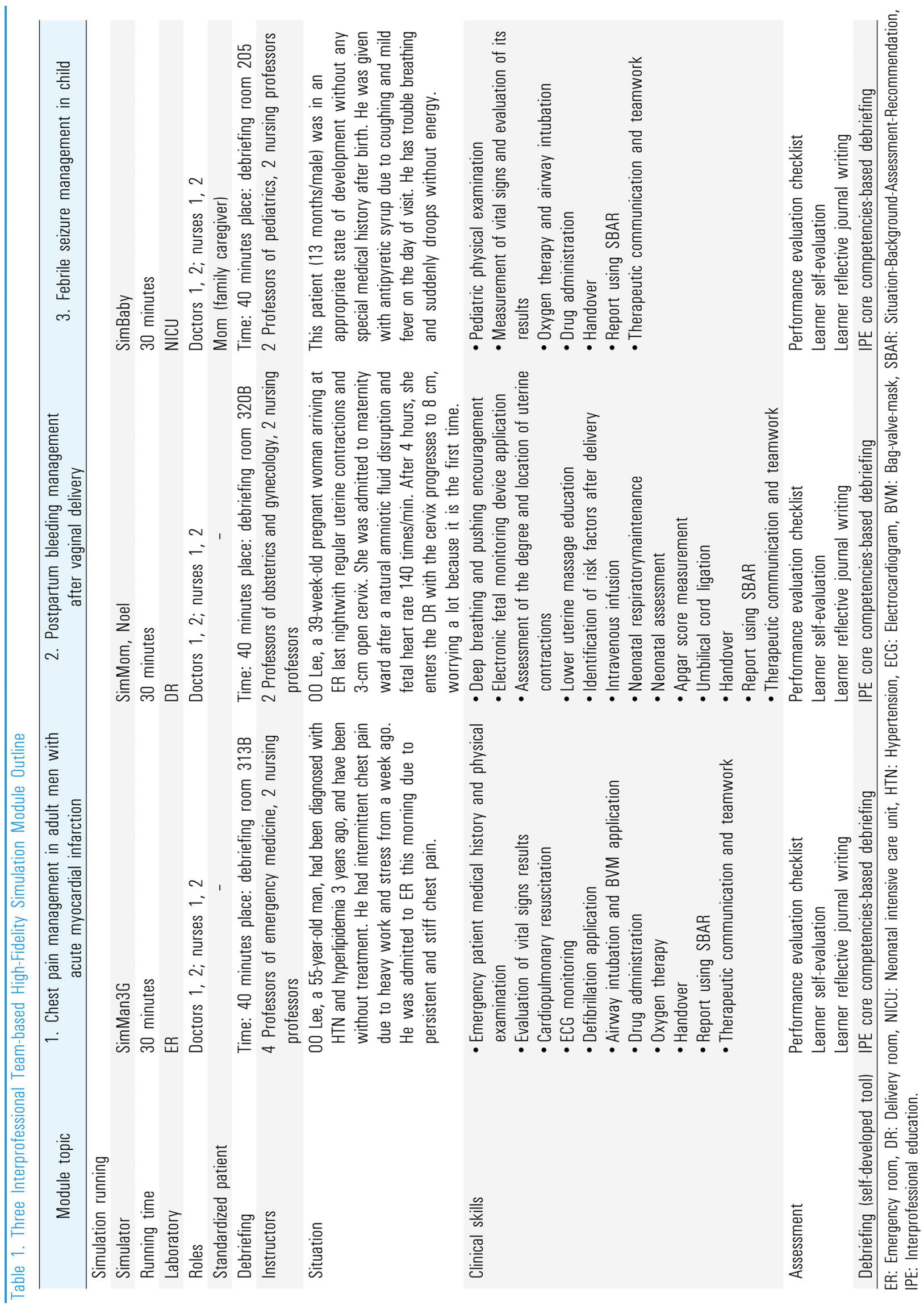


asked to write reflective journal about their perceptions of the impact of the IPSE. This was chosen because the use of reflective journals through the IPSE experience can enhance the experiential learning process by encouraging the participants to work through and re-evaluate their IPSE experience, which helps identify with their feelings and assess the experience in hindsight [13]. At the beginning and end of the IPSE program, they were also asked to write the keywords in order to identify their changes in attitudes towards each other's health professions after participating in the IPSE.

\section{Data analysis}

We used content analysis to analyze the reflective journal writing [14]. The data were first familiarized through reading and re-reading the texts. Initial codes across the entire data set were then generated in a systematic fashion with using an inductive analysis process. It allowed themes to emerge from the data. Themes were reviewed and then defined and named to ensure internal and external validity. The initial data analysis was conducted in Korean by the corresponding author, which was then reviewed in English, discussed, and refined with the research team until agreement was reached.

In order to find out nursing and medical students' changes in attitudes towards each other's health professions after participating in the IPSE, word cloud analysis was applied to keywords written before and after the IPSE program through word cloud generator (http://wordcloud.kr). It visualizes frequent keywords in a text where the size of the keywords represents their frequency, which is allowed to identify meaning and relationships easily through keyword size and color $[15,16]$.

\section{Ethical considerations}

Ethical approval was obtained from the Institutional Review Board of Ajou University (IRB approval no., AJIRB-SBR-SUR-20-125). All participants gave informed written consent prior to participating in this study.

\section{Results}

\section{Nursing and medical students' perceptions of the impact of the IPSE}

In terms of nursing and medical students' perspectives on their participation in the IPSE, three key themes emerged, and comprised five sub-themes (Table 2). The quotes reported in this study are representative of the sample and were translated from the Korean language texts.

\section{1) Positive experience}

Most nursing students reported positive experiences of participating in the IPSE. They said the IPSE experience helped them understand their own and medical professions' roles and responsibilities in providing health

\begin{tabular}{llr}
\hline Table 2. Nursing and Medical Students' & Perspectives on Participation in the Interprofessional Simulation-Based Education \\
\cline { 2 - 3 } \multicolumn{1}{c}{ Theme } & \multicolumn{1}{c}{ Sub-theme } \\
\hline Positive experience & \multicolumn{1}{c}{ Nursing students } & \multicolumn{1}{c}{ Medical students } \\
Positive learning outcomes & $\begin{array}{l}\text { Enhancing collaboration } \\
\text { Confidence in communication skills }\end{array}$ & \\
Benefits to patients of effective & $\begin{array}{l}\text { High quality of care } \\
\text { interprofessional practice }\end{array}$ & Patient safety \\
\hline
\end{tabular}


care. One nursing student wrote:

"I'm very interested $\cdots$ I actually practiced with medical students, managing the deteriorating patient together. It helped me understand what I and doctors do, when we were managing a patient' care together."

Medical students wrote good experiences to learn by doing in simulated clinical situations where patients are rapidly deteriorating, compared with lectures to learn interprofessional collaboration. They valued the simulation as it provided the opportunity for hands-on practice in a safe, realistic environment. One medical student wrote:

"It was amusing to me $\cdots$ I could practice patient care skills, particularly technical skills by doing in a controlled environment to save my patients."

\section{2) Positive learning outcomes}

Nursing and medical students recognized that the IPSE experience had a positive impact on collaboration enhancement. One nursing student wrote:

"Medical students' sense of empathy and respect led us to better collaboration in the team."

One medical student wrote:

"We need each other within the team, responding properly to the clinical situation where patients are rapidly getting worse and worse $\cdots$ because my team members filled what I lacked or missed in the situation ‥ I realized the importance of good teamwork to manage deteriorating patients."

Both groups expressed that the IPSE experience helped them improve their confidence in communication skills with team members in practice. One nursing student wrote:

"I'm more confidence in communicating effectively with medical students in providing patient care."

\section{3) Benefits to patients of interprofessional col- laborative practice}

Nursing and medical students perceived that IPSE experience helped them facilitate collaborative practice within the team to provide safe, quality health care and then improve patient care. One nursing student wrote:

"Due to various simulated clinical experiences, it promoted us working more effectively in providing better health care $\cdots$ I realized the importance of working collaboratively within the team for better patient outcomes."

One medical student commented:

"I perceived that collaboration with not only doctors but also nurses can provide better treatment and care to patients who are rapidly deteriorating $\cdots$ can improve their conditions."

Also, another nursing student stated:

“Considering the medical students' point of views helps me provide the patient with the safe, quality health care as a team."

\section{Changes in attitudes toward each other's health professions after IPSE experience}

All participants were asked to explore nursing and 
Fig. 2. Medical and Nursing Students' Attitudes Towards Each Other's Health Profession

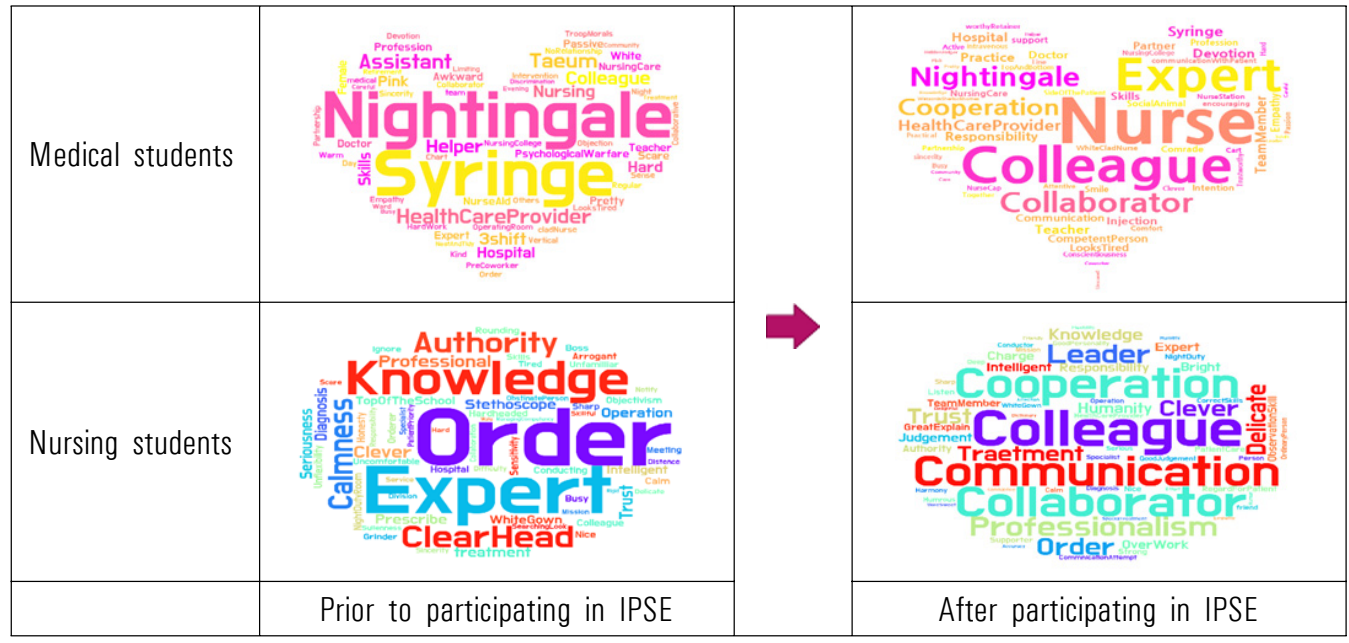

IPSE: Interprofessional simulation-based education.

medical students' attitudes toward each other's health professions before and after participating in the IPSE. Their changes in attitudes toward each other's health professions are displayed in Fig. 2. Before the IPSE experience, medical students perceived the nurse as nightingale, bullying culture called as "taeum" in Korean, syringe, three shifts, and assistants. Their attitudes toward nursing profession were changed to nursing professionals, colleagues, collaborators, and experts after participating in the IPSE.

Nursing students perceived the doctor as order, knowledge, expert, authoritative, and diagnosis before the IPSE experience. Their attitudes toward medical profession were changed to colleagues, collaboration, communication, leaders, and prescriptions after participating in the IPSE.

\section{Discussion}

This study provided undergraduate nursing and medical students' perceptions of the impact of the IPSE from a South Korean perspective. In this study, nursing and medical students who participated in the IPSE reported positive experience, positive learning outcomes and benefits to patients of interprofessional collaborative practice. Thus, this study confirms other similar Western work in IPSE area [17,18], which suggests that regardless of culture, the IPSE program can provide positive learning opportunities for undergraduate nursing and medical students to practice and develop interprofessional collaboration.

In this study, nursing and medical students provided examples of positive experiences. Most nursing students reported that IPSE experience helped them understand their own and medical professions' roles and responsibilities in providing health care. These findings support previous studies that demonstrated undergraduate health care professions students had greater understanding of their own and other health professions' roles in providing health care after the IPSE experience [17-20]. Thus, it suggests that the IPSE program needs to be incorporated into undergraduate health professions programs, which can transform undergraduate healthcare professions students' understanding of roles and responsibilities of their own and other healthcare profession before graduation. As a healthcare team member, it may contribute to interprofessional collaborative practice in 
providing health care.

As one of the positive experience examples, most medical students expressed the value of simulation activities incorporated in IPE. This finding might be explained by the course delivery of the medical discipline. Before this program, medical students did not experience HFS-based courses, although they are in year 3 of studies. Medical students in this study could learn how to work together with the team members by doing in simulated clinical situations. It allowed them to do hands-on practices in a safe, realistic environment $[8,9]$. Also, they could receive valuable and sincere feedback from nursing and medicine faculty facilitators and peers during a debriefing session [18]. Thus, this finding shows that the use of HFS in IPE provided the benefits of simulation for undergraduate medical and nursing students to learn and practice interprofessional collaboration through hands-on experience.

In this study, nursing and medical students reported collaboration enhancement as positive learning outcomes. Nursing students in this study had clinical practicums in a hierarchical hospital structure where physicians focus on biomedical treatment, saw nurses focus on medical treatment and follow the physician's perspective. A better rapport between nursing and medical students during the interprofessional teambased education led nursing students to perceive the sense of empathy and respect from medical students, which resulted in enhancing collaboration. Also, the team-based HFS education in each module promoted nursing and medical students' active involvement in patient care $[8,9]$. It led medical students to perceive the importance of good teamwork, which enhanced their collaboration.

Before the IPSE experience, nursing students in this study perceived the doctor as order, knowledge, expert, and authoritative, and medical students perceived the nurse as nightingale, bullying culture called "taeum" in Korean, and syringe. After the IPSE experience, both groups of students viewed each other as colleagues. It is believed that interprofessional team-based simulation education can promote collaborative teamwork, which might result in improving nursing and medical students' attitudes toward each other's health professions.

This study has several limitations. First, the data was collected only from one college of nursing and one school of medicine. Thus, the findings may not generalize to all colleges or schools of nursing and medicine in South Korea, limiting external validity. Second, the self-report reflective journal writing measured nursing and medical students' subjective evaluation of the IPSE experience. Thus, actual performance improvement in interprofessional collaboration could not be objectively concluded. Third, due to time and resource restraints, this study was the most feasible to provide a one-time IPSE program, so it tended to focus on the short-term (immediately post-IPSE) rather than explore the impact on clinical practice. Future research is needed to see how the reported benefits of IPSE training are reflected in clinical practice.

In conclusion, this study showed nursing and medical students perceived positive impact of the IPSE. Nursing students reported that the IPSE increased understanding of their own and medical profession's roles and responsibilities, enhanced collaboration, and increased confidence in communication skills with team members. Medical students also stated that IPSE allowed them to learn by doing in a safe, realistic simulated environments and enhanced collaboration and increased confidence in communication skills with team members. These positive findings may contribute to their future interprofessional collaborative practice after graduation, which may lead to help provide safe, quality health care in their workplace and ultimately improve patient outcomes. 


\section{ORCID:}

Woosuck Lee: https://orcid.org/0000-0002-1331-5541;

Miran Kim: https://orcid.org/0000-0001-5553-5334;

Yun Kang: https://orcid.org/0000-0001-9914-8969;

Yu-Jin Lee: https://orcid.org/0000-0003-4128-3222;

So Myeong Kim: https://orcid.org/0000-0002-6783-6107;

Janghoon Lee: https://orcid.org/0000-0003-4765-9948;

Soo-Jin Hyun: https://orcid.org/0000-0003-0196-850X;

Jihye Yu: https://orcid.org/0000-0002-8039-6580;

Young-suk Park: https://orcid.org/0000-0003-0311-2430

Acknowledgements: None.

Funding: No funding was obtained for this study.

Conflicts of interest: No potential conflict of interest relevant to this article was reported.

Author contributions: WL, MK, YK, YJL, SK, and JL: conception or design of the work; WL., MK, YK, YJL, SK, JL, SJH, JY, and YSP: data collection; YK and SK: data analysis and interpretation; WL, MK, YK, YJL, and SK: drafting the article; WL and YK: critical revision of the article and final approval of the version to be published.

\section{References}

1. Robben S, Perry M, van Nieuwenhuijzen L, et al. Impact of interprofessional education on collaboration attitudes, skills, and behavior among primary care professionals. J Contin Educ Health Prof. 2012;32(3):196-204.

2. World Health Organization. Framework for action on interprofessional education and collaborative practice (WHO/HRH/HPN/10.3). https://www.who.int/hrh/ resources/framework_action/en/. Published 2010. Accessed July 1, 2020.

3. Interprofessional Education Collaborative. Core competencies for interprofessional collaborative practice:
2016 update. Washington DC, USA: Interprofessional Education Collaborative; 2016.

4. Koo L, Layson-Wolf C, Brandt N, et al. Qualitative evaluation of a standardized patient clinical simulation for nurse practitioner and pharmacy students. Nurse Educ Pract. 2014;14(6):740-746.

5. Costello M, Prelack K, Faller J, Huddleston J, Adly S, Doolin J. Student experiences of interprofessional simulation: findings from a qualitative study. J Interprof Care. 2018;32(1):95-97.

6. Hood K, Cant R, Baulch J, et al. Prior experience of interprofessional learning enhances undergraduate nursing and healthcare students' professional identity and attitudes to teamwork. Nurse Educ Pract. 2014;14(2): 117-122.

7. Interprofessional Education Collaborative Expert Panel. Core competencies for interprofessional collaborative practice: report of an expert panel. https://www. ipecollaborative.org/core-competencies.html. Published 2011. Accessed July 1, 2020.

8. Reeves S, van Schaik S. Simulation: a panacea for interprofessional learning? J Interprof Care. 2012;26(3): 167-169.

9. Labrague LJ, McEnroe-Petitte DM, Fronda DC, Obeidat AA. Interprofessional simulation in undergraduate nursing program: an integrative review. Nurse Educ Today. 2018;67:46-55.

10. Decker SI, Anderson M, Boese T, et al. Standards of best practice: simulation standard VIII: simulation-enhanced interprofessional education (Sim-IPE). Clin Simul Nurs. 2015; $11(6): 293-297$.

11. Gough S, Hellaby M, Jones N, MacKinnon R. A review of undergraduate interprofessional simulation-based education (IPSE). Collegian. 2012;19(3):153-170.

12. Sanford PG. Simulation in nursing education: a review of the research. Qual Rep. 2010;15(4):1006-1011.

13. Rolfe G, Freshwater D, Jasper M. Critical reflection for 
nursing and the helping professions: a user's guide. Basingstoke, UK: Palgrave; 2001.

14. Norwood SL. Research essentials: foundations for evidence-based practice. Boston, USA: Pearson; 2010.

15. DePaolo CA, Wilkinson K. Get your head into the clouds: using word clouds for analyzing qualitative assessment data. TechTrends. 2014;58(3):38-44.

16. Cidell J. Content clouds as exploratory qualitative data analysis. Area. 2010;42(4):514-523.

17. Oxelmark L, Nordahl Amorøe T, Carlzon L, Rystedt H. Students' understanding of teamwork and professional roles after interprofessional simulation: a qualitative analysis. Adv Simul (Lond). 2017;2:8.

18. Roberts FE, Goodhand K. Scottish healthcare student's perceptions of an interprofessional ward simulation: an exploratory, descriptive study. Nurs Health Sci. 2018; 20(1):107-115.

19. Wang JN, Petrini M. Chinese health students' perceptions of simulation-based interprofessional learning. Clin Simul Nurs. 2017;13(4):168-175.

20. Fawaz M, Anshasi HA. Senior nursing student's perceptions of an interprofessional simulation-based education (IPSE): a qualitative study. Heliyon. 2019;5(10):e02546. 